\title{
One-dimensional nonequilibrium kinetic Ising models with branching annihilating random walk
}

\author{
Nora Menyhárd \\ Research Institute for Solid State Physics, H-1525 Budapest,P.O.Box 49, Hungary \\ E-mail:menyhard@power.kfki.szfki.hu
}

\begin{abstract}
Nonequilibrium kinetic Ising models evolving under the competing effect of spin flips at zero temperature and nearest neighbour spin exchanges at $T=\infty$ are investigated numerically from the point of view of a phase transition. Branching annihilating random walk of the ferromagnetic domain boundaries determines the steady state of the system for a range of parameters of the model. Critical exponents obtained by simulation are found to agree, within error, with those in Grassberger's cellular automata.
\end{abstract}

PACS Numbers: 05.70.Ln, 05.50.+q 


\section{Introduction}

Nonequilibrium kinetic Ising models, in which the steady state is produced by kinetic processes in connection with heat baths at different temperatures have been widely investigated[ 1 ] Most of these studies, however, are concerned with the effects the nonequilibrium nature of dynamics might exert on phase transitions driven by temperature.

A different line of investigating nonequilibrium phase transitions has been via branching annihilating random walk (BAW) processes. Here particles chosen at random carry out random walk (with probability $p$ ) with annihilation upon meeting. The increase of particles is ensured through production of $n$ offsprings with probability $1-p$. It has been shown by Sudbury[2] , that the $n=2$ BAW in one dimension leads to extinction for any $p>0$. Otherwise a phase transition occurs for finite $\mathrm{p}$, which is in the same universality class as directed percolation (DP) if $n=o d d$, while the critical behavior is different for $n=$ even [3 ].

Grassberger et al.[4 ] studied probabilistic cellular automata models in one dimension involving the processes $k \rightarrow 3 k$ and $2 k \rightarrow 0$ ( $k$ stands for kink), very similar to BAW with $n=2$. These models, however, do show a phase transition and both time-dependent and steady-state simulations have resulted in non-DP values for the relevant critical exponents [5 ]. Quite recently, Jensen [6] has reported computer simulation data according to which the $n=4 \mathrm{BAW}$ is in the same dynamic universality class as Grassberger's cellular automata.

It is the aim of the present paper to introduce a class of general nonequilibrium kinetic Ising models (NEKIM) with combined spin flip dynamics at $T=0$ and spin exchange dynamics at $T=\infty$ in which, for a range of parameters of the model, Grassberger-type transition takes place. The advantage over the cellular automaton formulation is that in NEKIM the rates of random walk, annihilation and kink-production processes can separately be controlled. This circumstance leads to the understanding of the $n=2$ BAW result which occurs in our model as a special case. Results

of computer simulations are presented for different critical exponents and scaling function from random as well as 1-kink initial states. 


\section{$2 \quad$ The model}

The model we will investigate here is a one-dimensional kinetic Ising model evolving by a combined spin-flip and spin-exchange dynamics with the restriction that spin flip transition probabilities satisfy detailed balance for the equilibrium state of the (ferromagnetic) Ising model at temperature $T=0$ while the spin exchanges are random $(T=\infty)$.

A general form of the Glauber spin-flip transition rate in one-dimension for spin $s_{i}$ sitting at site $i$ is [7] $\left(s_{i}= \pm 1\right)$ :

$$
W_{i}=\frac{1}{2 \Gamma}\left(1+\delta s_{i-1} s_{i+1}\right)\left(1-\frac{\gamma}{2} s_{i}\left(s_{i-1}+s_{i+1}\right)\right)
$$

where $\gamma=\tanh 2 J / k T$, with $J$ denoting the coupling constant in the Ising Hamiltonian $\Gamma$ and $\delta$ are further parameters which can also depend on temperature. There are three independent rates:

$$
\begin{aligned}
w_{\text {same }} & =\frac{1}{2 \Gamma}(1+\delta)(1-\gamma) \\
w_{\text {oppo }} & =\frac{1}{2 \Gamma}(1+\delta)(1+\gamma) \\
w_{\text {indif }} & =\frac{1}{2 \Gamma}(1-\delta),
\end{aligned}
$$

where the suffices same etc. indicate the three possible neighborhoods of a given $\operatorname{spin}(\uparrow \uparrow \uparrow, \downarrow \uparrow \downarrow$ and $\uparrow \uparrow \downarrow$,respectively). In the following $T=0$ will be taken, thus $\gamma=1, w_{\text {same }}=0$ and $\Gamma, \delta$ are constants to be varied. In this limit the three best-known single spin flip kinetic Ising models correspond to the following choices of parameters :

a). Glauber model $[8]: \Gamma=1, \delta=0$

b). Metropolis model[9]: $\Gamma=2 / 3, \delta=-1 / 3$

c). Haake-Thol model[10 ]: $\Gamma=2, \delta=1$.

The spin-exchange transition rate of neighbouring spins, originally introduced by Kawasaki[7 ] to situations with conserved magnetization, can be given, for $\gamma=0$, as:

$$
w_{i i+1}=\frac{1}{2} p_{e x}\left[1-s_{i} s_{i+1}\right]
$$

where $p_{e x}$ is the probability of spin exchange. 
The transition probabilities (2)-(4) are responsible for basic elementary processes of kinks in the usually expected ferromagnetical ordering of Ising spins. A kink separating two domains can carry out random walks with probability

$$
p_{\text {rw }} \propto 2 w_{\text {indif }}=\frac{1}{\Gamma}(1-\delta)
$$

while two kinks getting into neighbouring positions will annihilate with probability

$$
p_{\text {an }} \propto w_{\text {oppo }}=\frac{1}{\Gamma}(1+\delta)
$$

$\left(w_{\text {same }}\right.$ is responsible for creation of kink pairs inside of ordered domains at $T \neq 0)$.

In case of the spin exchanges, which also act only at phase boundaries, the process of main importance here is that a kink can produce two offsprings by the next time step with probability

$$
p_{k \rightarrow 3 k} \propto p_{e x}
$$

The abovementioned three processes compete, and it depends on the parameters $\Gamma, \delta$ and $p_{e x}$ what the result of this competition will be. It is important to realize that the process $k \rightarrow 3 k$ can develop into propagating offspring production only if $p_{r w}>p_{a n}$, i.e. the new kinks are able to travel on the average some lattice points away from their place of birth an can thus avoid immediate annihilation. It is seen from the above definitions that $\delta<0$ is necessary for this to happen. In the opposite case the only effect of the $k \rightarrow 3 k$ process on the usual Ising kinetics is to soften domain walls. As a matter of fact, in earlier investigations (though from a different point of view) of the competing Ising kinetics in one dimension the choices of parameters correspond to the latter situation [11].

\section{Phase boundary, critical exponents}

We have considered a simplified version of the above model by keeping only two parameters instead of three by imposing the condition

$$
p_{k \rightarrow 3 k}=1-\left(p_{r w}+p_{a n}\right) \text {. }
$$


In the plane of parameters $p_{e x}$ and $1-\delta=\frac{2 p_{r w} / p_{a n}}{1+p_{r w} / p_{a n}}$ the phase diagram shown on Fig.1. has been obtained by computer simulation. The initial state has been random with zero average magnetization . Application of our nonequilibrium rule then corresponds to quenching from $T=\infty$ to $T=0$ with subsequent exchanges $(T=\infty)$ at each time step. The line of phase transitions separates two kinds of steady states reached by the system for large times: in the Ising phase the system orders while the active phase is disordered from the point of view of the underlying spins. The cause of disorder is the steadily growing number of kinks with time. Kink-multiplication is here a branching process, as explained above, in a sense similar to directed percolation while in the usual case of Ising phase transition with Glauber kinetics kinks are created pairwise inside of ordered domains at nonzero temperature.

Exponent $\alpha$, used for determining the phase boundary, as well as other exponents occuring below are defined in the framework of the scaling considerations presented by Grassberger [5]. The branching increase of kinks becomes obvious when starting from a single kink state at $t=0$. The scaling form for the density $\rho(x, t, \epsilon)$ of the kinks has been supposed in the form $[5]$

$$
\rho(x, t, \epsilon) \propto t^{-\alpha} \phi\left(\epsilon x^{1 / \nu_{\perp}}, \epsilon t^{1 / \nu_{\|}}\right)
$$

Here $\epsilon$ measures the deviation from the critical probability at which the branching transition occurs, $\nu_{\perp}$ and $\nu_{\|}$are exponents of coherence lengths in space and time directions, respectively. $\phi(a, b)$ is analytic near $a=0$ and $b=0$. Using (10) the following relations can be deduced.

The average particle number $N(t, \epsilon)$ grows in the active phase as

$$
N(t, \epsilon) \propto t^{\eta} \psi\left(\epsilon t^{\left.1 / \nu_{\|}\right)} .\right.
$$

The RMS size of the cluster growing from the single-kink initial configuration is given by

$$
<(x-<x(t)>)^{2}>^{1 / 2} \propto<x_{\max }(t)-x_{\min }(t)>\propto t^{Z / 2}
$$

where $Z=2 \nu_{\perp} / \nu_{\|}$.

When starting from a random initial state the exponent $\beta$ characterizes the growth of the average kink density in the active phase:

$$
\rho(\epsilon)=\lim _{t \rightarrow \infty} \rho(x, t, \epsilon) \propto \epsilon^{\beta}
$$


while the decrease of density at the critical point is given by

$$
n(t) \propto t^{-\alpha} .
$$

The exponents are connected by scaling laws: $\beta=\nu_{\|} \alpha, \alpha=Z / 2-\eta$.

The phase boundary shown on Fig1. has been identified using (14). It is worth noting that the critical point marked on the phase boundary has been chosen in a region where the width of the critical region is relatively small. The rest of the critical exponents have been measured only around this point $: \frac{1}{\Gamma_{c}}=.35, \delta_{c}=-.4 \pm .01$ and $\left(p_{e x}\right)_{c}=1-\frac{2}{\Gamma_{c}}=.3 \pm .01$. The deviation from the critical point has been chosen in the direction of $\delta$.

The critical region is especially wide near the two ends of the curve. Moreover, near $p_{e x}=1.0$ where we get close to $\delta=0$ (Glauber case), it is very hard to determine $\alpha$ : the exponent grows slowly with time from a nearly zero value at small times. Several runs with different values of the parameters also without the restriction (9), have been performed in this Glauber limit all showing that the $\delta=0$ case for all $\Gamma, p_{e x}$ values, remains Ising-like: the exponent $\alpha$ tends to the value $\alpha=.5$ for large times. The accuracy of our simulations does not allow us to make a quite definite statement, nevertheless it seems very likely that the asymptote of the phase boundary is $\delta=0$ for $p_{e x}=1.0$. Therefore the conjecture that $p_{r w}>p_{a n}$ is a necessary condition for a BAW-type phase transition to occur seems to be supported by the present simulations.

The value of $\alpha$ provided by our simulations agrees, within error, with that obtained by Grassberger $[4,5]$ in a one -dimensional elementary cellular automaton model with special added noise $p$. The same is true also for the rest of the critical exponents reported below. Fig.2 shows the density of kinks in the active phase for different values of the deviation from the critical point $\epsilon=\left|\delta-\delta_{c}\right|$, starting from random initial states. At $\epsilon=0$ the power law behaviour (14), is seen with $\alpha=.27 \pm .02$. $\beta$ has been obtained directly, according to eq.(13), by taking the level-off values of $n(t)$, some of which can be read off from Fig.2. Though this is known to be a fairly inaccurate way to determine $\beta$ [5], we have found -though only over one decade in $\epsilon$ - the reasonable value $\beta=.8 \pm .08$.

On Figs. 3 through 5 results of simulations starting from one-kink initial states are seen. Data for $N(t, \epsilon)$ at $\epsilon=0$ are presented on Fig.3; the straight line on the $\log$ - $\log$ plot leads to $\eta=.30 \pm .02$. Fig. 4 shows the growth of the average cluster size, eq.(10), again on $\log -\log$ scale, yielding $Z / 2=.57 \pm .01$. Supposing the scaling law $\beta=\nu_{\|} \alpha$ to be valid we get $\nu_{\|}=2.9$. 
Using the values obtained for the exponents, we have checked the consistency of the data within the scaling framework by measuring the scaling form, eq.(11). The result is seen on Fig.5 for some values of $\epsilon$, nevertheless also only inside of a decade, because of computational difficulties. The second scaling law : $\alpha=Z / 2-\eta$ is also fulfilled with the exponents found in our simulations.

\section{Connection with BAW}

The definition of BAW is the following. The process is taking place in two steps: a). a particle chosen at random moves to a nearest neighbour place also randomly chosen, with probability $p$ and b). a particle chosen at random produces $\mathrm{n}$ offsprings on neighbouring sites with probability $1-p$. If any of the neighbouring places in question is already occupied then annihilation takes place. The $k+k \rightarrow 0$ process is prescribed to occur with probability unity. As mentioned in the introduction, no phase transition takes place in the $n=2$ case.

We can bring into relation the $n=2 \mathrm{BAW}$ in $1 \mathrm{~d}$ and NEKIM by noticing that in the BAW rule the transition probability for annihilation is unity. From eq. (7) $p_{a n}=1$ leads to $\Gamma=(1+\delta)$ giving, with eq.(6), $p_{r w}=\frac{1-\delta}{1+\delta}$. From the trivial correspondence

$$
\begin{aligned}
& p_{r w} \cong p \\
& p_{k \rightarrow 3 k} \cong 1-p
\end{aligned}
$$

while changing $p$, the parameters of the corresponding NEKIM models take the values from $\delta=1, p_{\text {ex }}=1, \Gamma=2$ at $p=0$ through values $\delta>0, p_{\text {ex }}<1$, $\Gamma<2$ to $\delta=0, p_{e x}=0, \Gamma=1$ at $p=1$. In these regions, however, no phase transition has been found to take place, all steady states are Ising-like.

\section{Discussion}

Non-thermal phase transition has been found in a family of one- dimensional kinetic Ising models evolving by a combined spin-flip and spin-exchange dynamics. The single spin flip transition rate is supposed in its most general form. It contains three parameters of which the temperature is fixed at 
zero where the remaining two control the probability of random walk and annihilation. The nonzero transition rate of spin exchange leads to $k \rightarrow 3 k-$ type kink production and provided RW overwhelms annihilation, increasing rate of kink production results in a phase transition from Ising-like to active phase. Numerical simulations have given evidence that the model is in the same universality class as Grassberger's cellular automata models[4,5], where a certain stochastic element (with probability p) of a simple 1d CA rule drives the transition. For $p=0$ the rule is deterministic and the steady state is infinitely degenerate, when starting from a random initial situation the system settles in one of these states after a few iteration steps. The corresponding kinetic Ising model is the Haake-Thol model in the $T \rightarrow 0$ limit mentioned in section 2. Increasing $p$ up to $p_{c}$ in the CA model, could correspond to a line on our phase diagram (Fig 1) starting at the origin and ending at some intermediate point of the transition line. It would be difficult, however, to make a closer (quantitative) correspondence between the two kinds of models since in CA this single parameter $\mathrm{p}$ is responsible for random walk as well as $k \rightarrow 3 k$ in a complicated,nonlinear way. To find correspondence between our model and the two offspring BAW is more straightforward. For this aim restriction (9) had to be lifted since $p_{a n}=1$ is taken in BAW rules and then eq (9) would lead to negative probabilities. According to the correspondence between BAW and NEKIM it is clear that a necessary condition to find a phase transition is $p_{a n}<1$.

\section{References}

1. Rácz Z and Zia K P Phys.Rev.E 49139 (1994) and references therein

2. Sudbury A. Ann. Probab. 18581 (1990)

3. Jensen I J.Phys. A:Math. Gen. 263921 (1993)

4. Grassberger $P$, Krause $F$ and von der Twer $T$ J.Phys.A:Math.Gen.17(1984)L105

5. Grassberger P J.Phys. A:Math.Gen. 22 (1989) L1103

6. Jensen I University of Melbourne Preprint, May 1994 
7. see e.g. Kawasaki K: Phase Transitions and Critical Phenomena,Vol.2., ed.Domb $C$ and Green $M S$ (New York: Academic, 1972) p.443

8. Glauber R J J.Math.Phys.4 (1963) 191

9. Metropolis $N$, Rosenbluth $A W$, Rosenbluth $M N$, Teller $A H$ and Teller E,

J.Chem.Phys. 21 (1953) 1087

10. Haake F and Thol K, Z.Physik B: Condensed Matter,40 (1980) 219

11. Droz $M$, Rácz $Z$ and Schmidt J

Phys.Rev. A39 (1989) 2141 


\section{Figure captions}

Fig 1 Phase diagram of the two-parameter model. The phase boundary has been obtained by measuring $n(t)$, the density of kinks, starting from a random initial distribution and locating the phase transition points by $n(t) \propto t^{-\alpha}$ with $\alpha=.27 \pm .04$. Typically the number of lattice points has been $l=2000$ and averaging over 500 independent runs has been performed. Checks for unimportance of finite size effects have been carried out. The dotted line with arrow indicates the critical point and the direction of deviation from it along which further critical characteristics have been determined.

Fig 2 Density of kinks as a function of time on a log-log plot for different values of $\epsilon=\left|\delta-\delta_{c}\right|$, where $\delta_{c}=-.4 \pm .01$ is the critical value of parameter $\delta$ at the chosen critical point. $p_{e x}$ is kept fixed at its critical value. Lattice sizes : 2000-8000, number of independent runs :400-800.

Fig 3 The growth of the average particle number at the critical point when starting from a single-kink initial state. The number of independent runs in the averaging: 900. The scale is double logarithmic.

Fig 4 Average distance between rightmost and leftmost kinks at the critical point on a double logarithmic scale. Average over 900 independent runs has been carried out.

Fig 5 Scaling function $\psi\left(\epsilon t^{1 / \nu_{\|}}\right)$of eq.(11) for different values of $\epsilon$. Initial state: single-kink, number of independent runs in averaging : 500. 